\title{
Prevalence of Hypertension and Associate Risk Factors among Workers at Hawassa University, Ethiopia: An Institution Based Cross Sectional Study
}

Atkilt Esaiyas*, Tesfaye Teshome and Dejene Kassa

College of Medicine and Health Sciences, Hawassa University, Ethiopia

\begin{abstract}
Background: Hypertension is a serious public health concern which alone is estimated to cause 7.5 million deaths $(12.8 \%$ of all causes of death) per year and it doubles the risk of many cardiovascular diseases.

Objectives: To assess the prevalence of hypertension, the associated risk factors and knowledge plus practice related to hypertension among workers at Hawassa University, Ethiopia.

Materials and methods: The study was conducted in January-March, 2014. Using simple random sampling technique 620 participants were selected. Data were collected by structured questionnaire. Data were analyzed using SPSS version 16 computer software.

Result: The response rate of the study was $99.6 \%$. Majority, $61.7 \%$ (376), of the respondents were male and $47.8 \%$ (272) were aged 25-34 years. Among respondents $19.7 \%$ (122) were found to be hypertensive either by systolic or diastolic blood pressure out of which $44 \%$ (54) were found hypertensive by both. Out of 122 hypertensive persons, $36.9 \%$ (45), did not know their hypertension status. Some $31 \%(192)$ of the respondents had a BMI score of 25 and above out of which $21.4 \%$ (41) were obese. Respondents whose BMI measures ranged from 25-29.99 and those who were 30 years old and above were at increased risk of hypertension $A O R=3.8(95 \% \mathrm{Cl} 1.22-12)$ and $\mathrm{AOR}=3.90(95 \% \mathrm{Cl} 1.10-14.01)$, respectively.
\end{abstract}

Conclusion: Prevalence of hypertension among the study subjects was significantly high and many of them didn't know their hypertension status. The level of hypertension related knowledge and practice of protective healthy life style were very low calling for urgent intervention.

Keywords: Body-mass-index; Waist-circumference; Hypertension; Obesity; Ethiopia

\section{Background}

Hypertension is clinically defined as a blood pressure of $140 / 90$ $\mathrm{mmHg}$ or more, based on at least two readings on separate occasions [1]. Non-communicable diseases (NCDs) which include hypertension are reported to be the leading causes of death globally, killing more people every year than all other causes of death together [2]. It is estimated that Hypertension alone causes 7.5 million deaths $(12.8 \%$ of all causes of death) per year and it increases the risk of cardiovascular diseases such as stroke, congestive heart failure, coronary heart diseases, renal failure, and peripheral arterial diseases by double [3].

Unlike the popular opinion, available data shows that nearly $80 \%$ of NCD deaths occur in low and middle-income countries [2]. SubSaharan Africa (SSA) has a disproportionate burden of both infectious and chronic diseases compared to other world regions. For Sub-Saharan Africa the current disease estimates are only based on limited data, but projections indicate that the existing demographic and epidemiologic transitions increase in non-communicable diseases (NCDs) [4].

In Ethiopia, according to reports by the Federal Ministry of Health hypertension accounted for 1.4 percent of all deaths in 2000/01, making it the $7^{\text {th }}$ leading cause of death in the country for the year [5]. According to $2014 \mathrm{WHO}$ report also Ethiopia lost 691,000 lives in the year of which $30 \%$ was due to NCDs [6].

Healthy work force is essential in the context of optimal productivity and enhanced competitiveness. Thus several studies explored the problem of hypertension amongst different groups of workers in India, Brazil and other countries [7,8]. Generally the information on hypertension in Ethiopia is limited to our knowledge and there is no previous data on hypertension prevalence and associated factors among occupational groups conducted in Ethiopia.

\section{Methods}

\section{Study design and setting}

A cross sectional study design was employed from January, 2014 to March, 2014. The study was conducted in Hawassa University: Main, Agriculture, Wendo Genet, Awada and Referral campuses. Hawassa is located $273 \mathrm{~km}$ south of Addis Ababa and is the regional capital of Southern Nations Nationalities and Peoples Region. The University has nearly 7,000 permanent and other contractual workers. The study subjects were selected from each department (administrative, academic and hospital) based on simple random sampling technique from lists obtained from all Colleges and the Institute of Technology human resource offices. Using EPI INFO version 6.04 sample sizes was calculated based on single population proportion. Based on this 622 staff members were selected out of which 620 respondents were obtained.

*Corresponding author: Atkilt Esaiyas, College of Medicine and Health Sciences Hawassa University, Ethiopia, Tel: +251911714976; E-mail: atuesu@gmail.com

Received September 08, 2017; Accepted December 28, 2017; Published January 11, 2018

Citation: Esaiyas A, Teshome T, Kassa D (2018) Prevalence of Hypertension and Associate Risk Factors among Workers at Hawassa University, Ethiopia: An Institution Based Cross Sectional Study. J Vasc Med Surg 6: 354. doi 10.4172/2329-6925.1000354

Copyright: ( $\odot 2018$ Esaiyas A, et al. This is an open-access article distributed under the terms of the Creative Commons Attribution License, which permits unrestricted use, distribution, and reproduction in any medium, provided the original author and source are credited. 
Citation: Esaiyas A, Teshome T, Kassa D (2018) Prevalence of Hypertension and Associate Risk Factors among Workers at Hawassa University, Ethiopia: An Institution Based Cross Sectional Study. J Vasc Med Surg 6: 354. doi: 10.4172/2329-6925.1000354

Page 2 of 6

\section{Data collection}

All data were collected following a well-developed, structured questionnaire consisting of close ended questions. The questionnaire covered demographic data: Knowledge and practice of the participants and anthropometric measurement space filled by experienced nurse data collectors. Pilot study was carried out at Hawassa College of Teacher's Education to evaluate the validity of the questions before actual data collection. The English version of the questionnaire was translated into Amharic, the official language of the study area and was back-translated to English to ensure consistency. Confidentiality of the findings was strictly maintained on all collected data.

A digital scale was used for weight measurement. Standardized height measuring device was used for height measurements. Waist circumference was measured with a soft tape on standing subjects midway between the lowest rib and the iliac crest. A private area was chosen for a tape measurement. Application of the tension tape over the marked point making sure that the tape was horizontal across the back; and making the recording at the point of normal expiration.

BP was measured with a regularly serviced mercury sphygmomanometer. At the subject's first BP assessment, BP was measured while both arms were placed on the table with the palm facing upwards. The artery position mark was aligned with the brachial artery. The subject remained resting for 5 minutes at the data collection center and it was made sure that they didn't take caffeine, alcohol, and had physical activity/exercise for at least 30 minutes earlier the BP measurement. The second BP measurement was taken after 10 minutes from the first measurement. Employees who worked in the University as a permanent worker during the data collection period and were willing to take part in the study were included while daily contract workers, severely sick during the data collection period, employees who are on annual/study leave and out of town during the data collection period, pregnant women during the data collection period and women within 45 days after delivery were excluded from the study.

\section{Data quality assurance}

Questionnaires were checked for completeness, accuracy, clarity and appropriateness on a daily basis by the investigators at the time of data collection. Experienced nurses were selected for the data collection procedure. Training was given to data collectors and facilitators by the investigators and guest senior internist to increase the quality of data.

\section{Ethical issues}

Ethical clearance was obtained from the Institutional Review Board of Hawassa University College of Medicine \& Health Sciences before the start of the research and all ethical requirements were adhered to.

\section{Results}

Data from a total of 620 respondents who completed the structured questionnaire were eligible for analysis, providing an overall response rate of $99.6 \%$. The majority, $48.4 \%$ (300) and $21.3 \%$ (132) of the respondents participated from the Main Campus and College of Medicine and Health Sciences, respectively. The mean age of the respondents was 33.9 years $(\mathrm{SD}=9.0)$ and those $25-34$ years old dominated the sample, accounting, 47.8\% (272). Out of 609 respondents who reported their marital status, $60 \%$ (365) were married, $36.5 \%$ (222) were single and $2 \%(12)$ were divorced. Out of this study's 620 rrespondents, $50.9 \%$ (312) were protestant and $43.2 \%$ (265) were Orthodox Christians. Details of socio-demographic characteristics of the respondents are presented in Table 1.

\begin{tabular}{|c|c|c|}
\hline Characteristics & Frequency & $\%$ \\
\hline \multicolumn{3}{|l|}{ Campus } \\
\hline Main & 300 & 48.4 \\
\hline Medical & 132 & 21.3 \\
\hline Wendo Genet & 90 & 14.5 \\
\hline Agriculture & 60 & 9.7 \\
\hline Awada & 38 & 6.1 \\
\hline \multicolumn{3}{|l|}{ Age (years) } \\
\hline$<=24$ & 62 & 10.9 \\
\hline $25-34$ & 272 & 47.8 \\
\hline $35-44$ & 151 & 26.5 \\
\hline $45-54$ & 63 & 11.1 \\
\hline$=>55$ & 21 & 3.7 \\
\hline \multicolumn{3}{|l|}{ Sex } \\
\hline Male & 376 & 61.7 \\
\hline Female & 233 & 38.3 \\
\hline \multicolumn{3}{|l|}{ Level of Education } \\
\hline$\leq$ Grade 12 complete & 175 & 28.5 \\
\hline Diploma & 203 & 33.1 \\
\hline First degree & 179 & 29.2 \\
\hline$\geq$ Second degree & 57 & 9.3 \\
\hline \multicolumn{3}{|l|}{ Occupation } \\
\hline Health Professional & 59 & 9.9 \\
\hline Adm-staff & 472 & 78.9 \\
\hline Academic Staff & 67 & 11.2 \\
\hline \multicolumn{3}{|c|}{ Monthly Income (ETB) } \\
\hline$<1000$ & 131 & 30.2 \\
\hline $1000-3000$ & 179 & 41.2 \\
\hline $3001-5000$ & 66 & 15.2 \\
\hline$>5000$ & 58 & 13.4 \\
\hline
\end{tabular}

Table 1: Socio-demographic and Economic characteristics.

Only 70\% (434) responses were obtained in relation to information about income of the household. The reported median monthly income of the households in this study was 1500 (IQR=1500) Ethiopian birr.

\section{Prevalence of Hypertension}

The prevalence of hypertension in this study refers to the number of respondents whose average value of either systolic blood pressure 140 or above $\mathrm{mmHg}$ or diastolic blood pressure measured $90 \mathrm{mmHg}$ or above. Therefore, a person whose average BP $\geq 140 / 90 \mathrm{mgHg}$, was classified hypertensive. The number of cases satisfying the above definition was divided by the total number of all individuals whose blood pressure was measured at least twice and then, multiplied by one hundred.

Out of the 620 respondents, $19.7 \%$ (122) were found to be hypertensive either by systolic or diastolic blood pressure out of which $44 \%$ (54) were found hypertensive both by systolic and diastolic blood pressure. Out of 122 hypertensive persons, 23\% (28) reported that they have already known their hypertensive status, while $40 \%$ (49) said that they never been hypertensive, and the remaining $36.9 \%$ (45) did not know their hypertension status (Figure 1). Among the study's 604 respondents, $22.7 \%$ (137), responded that they have hypertension case among their family members while $77.3 \%$ (467) responded that there is no hypertension case in their family.

\section{Respondents' Knowledge about Hypertension and Related Practices}

\section{Knowledge levels}

Respondents' knowledge levels about hypertension could influence 
Citation: Esaiyas A, Teshome T, Kassa D (2018) Prevalence of Hypertension and Associate Risk Factors among Workers at Hawassa University, Ethiopia: An Institution Based Cross Sectional Study. J Vasc Med Surg 6: 354. doi: 10.4172/2329-6925.1000354

Page 3 of 6

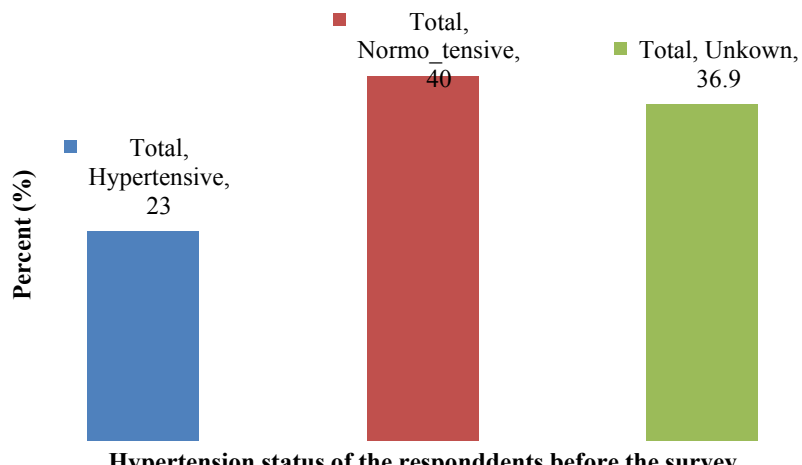

Hypertension status of the responddents before the survey

Figure 1: Reported hypertension status of the respondents who tested hypertensive during the study $(n=122)$

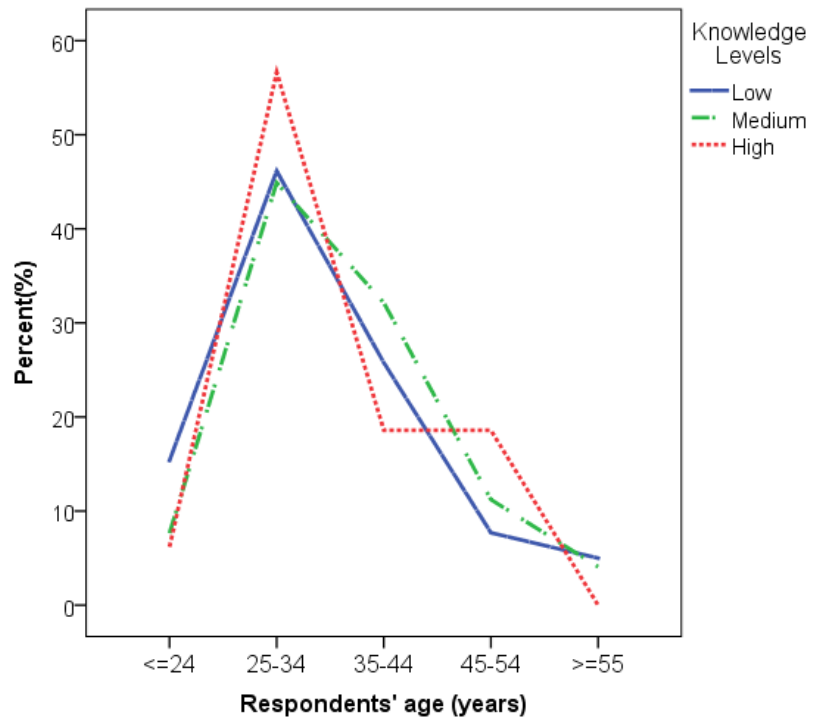

Figure 2: Respondents' ages compared with their hypertension-related knowledge levels $(n=620)$.

their preventive and control actions. The knowledge of the respondents who participated in this study was described based on individual items using frequencies and corresponding percentages. The overall knowledge level was also assessed by computing scores for correct responses of all relevant items into a single composite variable. This approach was used to perform further analyses about respondents' hypertension-related knowledge.

The overall proportion of correct responses to questions that assessed knowledge of the respondents about hypertension ranged from a minimum of $53.4 \%$ to $97.7 \%$.

The overall knowledge of the interviewees was computed from the 9 knowledge items and categorized into a single composite variable with three ordinal response options. There were $46.1 \%$ (286) of the respondents with "low level" knowledge about hypertension, of them had "medium level" knowledge, and 19\% (118) had "high level" knowledge (Figure 1). The median and inter quartile range of knowledge score were 7 and 3 out of possible 9 points.

Associations of selected socio-demographic characteristics of the respondents and their overall level of knowledge about hypertension were examined using Pearson Chi-square tests. Outputs of association between the levels of knowledge and socio-demographic variables are presented in Table 2. Age had a significant effect on the respondents' knowledge levels about prevention and control of hypertension. Out of 113 participants who had high knowledge levels, 64 (56.6\%) were aged 25-34 years (Figure 2). Only $44.1 \%(n=120)$ of this age group out of 260 had low levels of knowledge about hypertension compared to other age categories. In this study, level of education of the respondents, occupation, age and monthly income of the family were significantly associated with the level of the respondents' knowledge about hypertension $(\mathrm{p}<0.001)$. However, knowledge levels did not vary with sex and marital status of the study participants ( $>0.05)$ (Table 2).

\section{Practices related to hypertension}

Out of 588 respondents, $49.7 \%$ (292) had checked their blood pressure in the last one year and the remaining $50.3 \%$ (296) did not. Similarly, out of 591, 45.5\% (269) reported that they do some physical exercises with the frequencies ranging from once a day (14.7\%) to only 1-3 times per week (24.2\%). On the other hand, the majority $54.5 \%$ (322) never practiced any exercise. With regard to the undesirable behaviors, only $1.2 \%$ (7) and $11.8 \%$ (68) and of the respondents reported that they smoke cigarettes and drink alcohols, respectively.

\section{Anthropometric measurements}

Among the anthropometric measurements taken during the study; waist circumference and BMI which summarizes the height and

\begin{tabular}{|c|c|c|c|c|c|}
\hline \multirow[b]{2}{*}{ Variables } & \multicolumn{3}{|c|}{ Knowledge Categories } & \multirow[b]{2}{*}{$\mathbf{X}^{2}$} & \multirow[b]{2}{*}{$\mathbf{P}$} \\
\hline & Low & Medium & High & & \\
\hline \multicolumn{6}{|l|}{$\operatorname{Sex}(n=609)$} \\
\hline Male & 178 & 135 & 63 & & \\
\hline Female & 101 & 79 & 53 & 3.4 & 0.19 \\
\hline \multicolumn{6}{|l|}{ Age (years) $(n=569)$} \\
\hline$\leq 24$ & 40 & 15 & 7 & & \\
\hline $25-34$ & 120 & 88 & 64 & & \\
\hline $35-44$ & 67 & 63 & 21 & 30.3 & 0.001 \\
\hline $45-54$ & 20 & 22 & 21 & & \\
\hline$\geq 55$ & 13 & 8 & 0 & & \\
\hline \multicolumn{6}{|c|}{ Highest education attended $(n=614)$} \\
\hline$\leq$ Grade 12 & 105 & 57 & 13 & & \\
\hline Diploma complete & 94 & 77 & 32 & 50.7 & 0.001 \\
\hline First degree complete & 72 & 59 & 48 & & \\
\hline Second degree and above & 12 & 22 & 23 & & \\
\hline \multicolumn{6}{|l|}{ Monthly income $(n=434)$} \\
\hline$<1000$ & 80 & 42 & 9 & & \\
\hline $1000-3000$ & 72 & 73 & 34 & & \\
\hline $3001-5000$ & 19 & 25 & 22 & 43.9 & $<0.001$ \\
\hline$>5000$ & 14 & 24 & 20 & & \\
\hline \multicolumn{6}{|l|}{ Occupation $(n=598)$} \\
\hline Health professional & 2 & 22 & 35 & 100 & $<0.001$ \\
\hline Administrative staff & 249 & 165 & 58 & & \\
\hline Academic staff & 23 & 20 & 24 & & \\
\hline \multicolumn{6}{|c|}{ History of hypertension in the family $(n=604)$} \\
\hline Yes & 42 & 9 & 36 & 16.5 & $<0.001$ \\
\hline No & 234 & 152 & 81 & & \\
\hline \multicolumn{6}{|l|}{ Marital status $(n=609)$} \\
\hline Single & 115 & 65 & 42 & 10.4 & 0.108 \\
\hline Married & 162 & 149 & 76 & & \\
\hline
\end{tabular}

Table 2: Association of knowledge levels and selected socio-demographic characteristics of the respondents, May 2014. 
Citation: Esaiyas A, Teshome T, Kassa D (2018) Prevalence of Hypertension and Associate Risk Factors among Workers at Hawassa University, Ethiopia: An Institution Based Cross Sectional Study. J Vasc Med Surg 6: 354. doi: 10.4172/2329-6925.1000354

Page 4 of 6

weight data have been tabulated below. One-fourth of males scored wait circumference of at least $94 \mathrm{~cm}$ and one third of the females scored waist circumference of at least $80 \mathrm{~cm}$. Over $192(31 \%)$ of the respondents had a BMI score of 25 and above out of which $41(21.4 \%)$ were obese (had a BMI exceeding or equal to 30.0).

The association of waist circumference and the systolic blood pressure was examined using a simple logistic regression model. The result showed that as the waist circumference increased by one centimeter, the SBP increased by $0.24 \mathrm{~mm}$ of mercury (95\%CI: 0.17 , $0.30)$.

The SBP of the respondents consistently increased as the BMI increased.

\section{Socio-demographic and economic predictors of hypertension among the study subjects}

Identification of factors that could explain prevention and control practices of hypertension at the household level was one of the core objectives of this study. There was no association between the hypertension status of the respondents and some sociodemographic characteristics $(p>0.05)$. These included: sex, marital status, occupation of the respondent and monthly household income (Table 3).

Association of practices and anthropometric measures of study subjects with their hypertension status was examined (Table 4). The output showed that, those who did not check their hypertension status over the last 12 months preceding the study were less likely to test positive for hypertension compared to those who did the checkups, $\mathrm{AOR}=0.49$ (95\%CI 0.29, 0.86). BMI was another independent factor that predicted hypertension. Respondents whose BMI measures ranged from 25-29.99 and those who were 30 years old and above were at increased risk of hypertension $\mathrm{AOR}=3.8$ (95\%CI 1.22-12) and $\mathrm{AOR}=3.90(95 \% \mathrm{CI} 1.10,14.01)$, respectively. On the other hand, performance of the physical exercise, consumption of alcohol and knowledge level about hypertension did not affect hypertension status of the respondents.

\section{Discussion}

This study revealed the following main findings. The prevalence of hypertension (blood pressure $\geq 140 / 90 \mathrm{mmHg}$ ) among the study population was $19.7 \%$, of which $44 \%$ tested hypertensive by both systolic and diastolic measures. Regarding the level of awareness of the respondents about hypertension, only $22 \%$ had high level of knowledge. About $49.7 \%$ of the participants reportedly said that they regularly check their BP and $45 \%$ perform exercises on a regular basis. Almost all (98.8\%) never smoked cigarettes and $88.2 \%$ did not take alcohol. About $31 \%$ of the respondents had a BMI measure of at least 25 . Age, taking regular BP checkups and BMI were among the factors identified to independently predict hypertension status of the study participants.

The reported hypertension prevalence of $19.7 \%$ in the study's sample is less than the finding reported by Mulenga and Siziya among academic staff from University of Zambia, which reported a prevalence of $40 \%$ [9]. The observed difference might be attributed to the reported level of exposure to the risk factors for hypertension. In the Zambian

\begin{tabular}{|c|c|c|c|c|}
\hline \multirow[t]{2}{*}{ Predictors } & \multicolumn{2}{|c|}{ Hypertension } & \multirow[t]{2}{*}{ COR $(95 \% \mathrm{Cl})$} & \multirow[t]{2}{*}{ AOR $(95 \% \mathrm{Cl})$} \\
\hline & Normal & Hypertensive & & \\
\hline \multicolumn{5}{|l|}{ Sex } \\
\hline Male & 284 & 92 & 1 & 1 \\
\hline Female & 28 & 28 & $0.42(0.27,0.67)^{*}$ & $0.57(0.30,1.08)$ \\
\hline \multicolumn{5}{|l|}{ Age (years) } \\
\hline$\leq 24$ & 58 & 4 & 1 & 1 \\
\hline $25-34$ & 231 & 41 & $2.6(0.89,7.50)$ & $2.2(0.59,7.89)$ \\
\hline $35-44$ & 113 & 38 & $4.9(1.70,14.30)^{*}$ & $4.58(1.17,17.89)^{*}$ \\
\hline $45-54$ & 36 & 27 & $10.9(3.5,33.6)^{*}$ & $7.35(1.65,32.79)^{*}$ \\
\hline$\geq 55$ & 16 & 5 & $4.5(1.10,18.9)^{*}$ & $2.17(0.33,14.39)$ \\
\hline \multicolumn{5}{|l|}{ Educational status } \\
\hline Grade 12 or less & 144 & 31 & 1 & 1 \\
\hline Diploma complete & 167 & 36 & $1.0(0.59,1.70)$ & $2.14(1.02,4.46)^{*}$ \\
\hline First degree complete & 144 & 35 & $1.1(0.66,1.9)$ & $1.87(0.81,4.36)$ \\
\hline Second degree or above & 38 & 19 & $2.3(1.2,4.6)^{*}$ & $2.46(0.63,9.67)$ \\
\hline \multicolumn{5}{|l|}{ Marital status } \\
\hline Single & 196 & 26 & 1 & 1 \\
\hline Married & 279 & 86 & $2.3(1.4,3.7)^{*}$ & $1.38(0.74,2.57)$ \\
\hline Divorced & 11 & 1 & $0.69(0.09,5.5)$ & $0.72(0.07,7.41)$ \\
\hline Widowed & 5 & 5 & $7.5(2 \cdot 0,27.8)^{*}$ & $5.3(0.94,29.94)$ \\
\hline \multicolumn{5}{|l|}{ Occupation $(n=857)$} \\
\hline Health professional & 50 & 9 & 1 & 1 \\
\hline Administrative staff & 376 & 96 & $1.4(0.67,2.9)$ & $1.89(0.65,5.57)$ \\
\hline Academic staff & 53 & 14 & $1.47(0.58,3.70)$ & $1.21(0.32,4.63)$ \\
\hline \multicolumn{5}{|l|}{ Monthly income (ETB) } \\
\hline$<1000$ & 106 & 25 & 1 & 1 \\
\hline $1000-3000$ & 138 & 41 & $1.3(0.72,2.20)$ & $0.78(0.39,1.54)$ \\
\hline $3001-5000$ & 53 & 13 & $1.04(0.49,2.20$ & $0.66(0.22,1.96)$ \\
\hline$>5000$ & 43 & 15 & $1.48(0.71,3.10)$ & $0.69(0.22,2.17)$ \\
\hline
\end{tabular}

*The test is significant at $\alpha<0.05$

Table 3: Socio-demographic and economic predictors of hypertension among Hawassa University staff, 2014. 
Citation: Esaiyas A, Teshome T, Kassa D (2018) Prevalence of Hypertension and Associate Risk Factors among Workers at Hawassa University, Ethiopia: An Institution Based Cross Sectional Study. J Vasc Med Surg 6: 354. doi: 10.4172/2329-6925.1000354

Page 5 of 6

\begin{tabular}{|c|c|c|c|c|}
\hline \multirow[t]{2}{*}{ Predictors } & \multicolumn{2}{|c|}{ Hypertension } & \multirow[t]{2}{*}{ COR $(95 \% \mathrm{Cl})$} & \multirow[t]{2}{*}{ AOR $(95 \% \mathrm{Cl})$} \\
\hline & Normal & Hypertensive & & \\
\hline \multicolumn{5}{|l|}{ Check their hypertension status } \\
\hline In the past six months & 146 & 56 & 1 & 1 \\
\hline In the past 12 months & 72 & 18 & $0.65(0.36,1.19)$ & $0.70(0.36,1.36)$ \\
\hline I didn't have checkups in the last 12 months & 255 & 41 & $0.42(0.27,0.66)^{\star}$ & $0.49(0.29,0.86)^{*}$ \\
\hline \multicolumn{5}{|l|}{ Perform physical exercises } \\
\hline Daily & 72 & 15 & 1 & 1 \\
\hline 1-3 times/week & 197 & 36 & $1.6(0.82,3.20)$ & $1.70(0.79,3.56)$ \\
\hline 4-6times/week & 34 & 5 & $0.71(0.24,2.10)$ & $0.76(0.24,2.43)$ \\
\hline Never & 202 & 60 & $1.10(0.59,0.93)$ & $0.94(0.46,1.89)$ \\
\hline \multicolumn{5}{|l|}{ Consume alcohol } \\
\hline Yes & 48 & 20 & 1 & 1 \\
\hline No & 415 & 91 & $0.53(0.29,0.93)^{*}$ & $0.54(0.29,1.01)$ \\
\hline \multicolumn{5}{|l|}{$\mathrm{BMI}$} \\
\hline$<18.5$ & 47 & 5 & 1 & 1 \\
\hline $18.5-24.99$ & 323 & 51 & $1.48(0.56,3.90)$ & $1.35(0.45,4.10)$ \\
\hline $25-29.99$ & 101 & 50 & $4.65(1.74,12.43)^{*}$ & $3.83(1.22,12.0)^{*}$ \\
\hline$\geq 30$ & 26 & 15 & $5.4(1.7,16.62)^{\star}$ & $3.93(1.10,14.01)^{*}$ \\
\hline \multicolumn{5}{|l|}{ Knowledge levels } \\
\hline Low & 209 & 47 & 1 & 1 \\
\hline Medium & 179 & 50 & $1.24(0.79,1.94)$ & $0.84(0.49,1.45)$ \\
\hline High & 110 & 25 & $1.01(0.59,1.73)$ & $0.61(0.32,1.19)$ \\
\hline
\end{tabular}

*The test is significant at $\alpha<0.05$

Table 4: Association of practices and anthropometric measures with hypertension status of the participants.

study, $22 \%$ smoke cigarettes and $63 \%$ consume alcohol which is very high compared to the current findings (only $1.2 \%$ smoked and $11.8 \%$ consumed alcohol).

The current study's prevalence is also lower compared to finding from a systematic review that involved 45 different studies conducted in West African countries, an overall prevalence ranging from $12 \%$ $68.9 \%$ was reported among different workforces [10]. Specifically, the prevalence of hypertension among health care, bank workers and civil servants ranged from $17.5 \%$ to $37.5 \%$. The prevalence of hypertension observed in this study is also lower compared to that reported among staff in Nigerian teaching hospital which was 36.6\% [11].

This study showed that respondents older than 35 years were over four folds more likely to be hypertensive compared to those at most 24 years of age. Similar findings about the overall role of age in increasing the risk of hypertension among university staff were reported from Zambia [9] and West Africa [10].

\section{Conclusions}

From this study it might be concluded that prevalence of hypertension among the study subjects is significantly high (19.7\%) and out of these $77 \%$ didn't know their hypertension status. This could be considered an alarming figure with potential consequences in not making the necessary adjustments in modifiable risk factors and monitoring the progression of the disorder. Moreover, the level of knowledge related on hypertension and practice of protective healthy life style also pose serious public health concern.

\section{Recommendation}

Based on the findings in this study we strongly recommend: wider scale, multicentre investigations with more parameters for better picture on the point. Aggressive work by all stakeholders in synergistic approach towards awareness creation regarding hypertension, promotion of healthy life style and improving health checkups among the community is also highly recommended.

\section{Acknowledgement}

The researchers would like to thank, Hawassa University, Vice President Office for Research and Technology Transfer, for the financial support obtained Ato Sintayehu Tadesse for the support during the initiation phase of the project, all the research assistants and respondents for their generous support for the success of the research.

\section{References}

1. Abdullahi AA, Amzat $\mathrm{J}$ (2011) Knowledge of hypertension among the staff of University of Ibadan, Nigeria. Journal of Public Health and Epidemiology 3: 204-209.

2. World Health Organization (2010) Global status report on non-communicable diseases. Italy.

3. Awoke A, Awoke T, Alemu S, Megabiawet B (2012) Prevalence and associated factors of hypertension among adults in Gondar, Northwest Ethiopia: a community based cross-sectional study. BMC Cardiovascular Disorders 12: 113.

4. Dalal S, Volmink J, Adebamowo C, Bajunirwe F, Njelekela M, et al. (2011) Non-communicable diseases in sub-Saharan Africa: what we know now. International Journal of Epidemiology 40: 885-901.

5. Getahun W. Gedif T. and Tesfaye F. (2010) Regular Khat (Catha edulis) chewing is associated with elevated diastolic blood pressure among adults in Butajira, Ethiopia: A comparative study. BMC Public Health 10: 390.

6. World Health Organization (2014) Non-communicable Diseases (NCD) Country Profiles 2014.

7. Divan V, Chauhan V, Panchal S, Bansal RK (2010) Prevalence of hypertension amongst workers of a fertilizer company in Surat district. Natl J Community Med 1:153-155.

8. Martinez MC, Latorre MR (2006) Risk factors for hypertension and diabetes mellitus in metallurgic and siderurgic company's workers. Arq Bras Cardiol 87: 424-432.

9. Mulenga D, Siziya S (2013) Prevalence and Correlates for Hypertension among Full-Time UNZA Academic Staff. Medical Journal of Zambia 40: 146-149. 
Citation: Esaiyas A, Teshome T, Kassa D (2018) Prevalence of Hypertension and Associate Risk Factors among Workers at Hawassa University, Ethiopia: An Institution Based Cross Sectional Study. J Vasc Med Surg 6: 354. doi: 10.4172/2329-6925.1000354

Page 6 of 6

10. Bosu WK (2015) The prevalence, awareness, and control of hypertension among workers in West Africa: a systematic review. Global Health Action 8: 22267
11. Funke O (2013) Blood pressure and body mass index among Jos University Teaching Hospital Staff. Transnational Journal of Science and Technology 3 : $67-83$ 